\title{
Cytological study of six Salvia species (lamiaceae) from the Hengduanshan Mountains region of China
}

\author{
Yang Zhiyun, Xun Gong* and Yuezhi Pan \\ Kunming Institute of Botany, Chinese Academy of Sciences, Kunming, Yunnan 650204, P. R. China
}

\begin{abstract}
Salvia is a genus with surprisingly diverse chromosome numbers. In this paper six species were cytologically studied from Hengduanshan Mountains region, where is one of two diversification centers of the genus. S. przewalskii var. przewalskii is a tetraploid with the chromosome number of $2 \mathrm{n}=4 \mathrm{x}=32$, other five species are the diploids with chromosome numbers of $2 n=2 x=16$. The basic chromosome number $x=8$ was inferred for all species studied, which was different from $x=7,11$ and 16 reported for species of the genus distributed in other regions. Several recent cytological studies on different groups from Hengduanshan Mountains region showed that only few polyploids infers that variation of karyotype structure at the diploid level seems to play a leading role and sympatric speciation via hybridization and polyploidization has played a minor role in speciation of some groups in Hengduanshan Mountains region.
\end{abstract}

Key words: chromosome; Hengduanshan Mountains; karyotype; Salvia; speciation

\section{INTRODUCTION}

The genus Salvia L., with about 900 species (MABBERLEy 1998), is probably the largest member of the family Lamiaceae. Species of the genus widely distributed in tropical and temperate areas of both Old and New World. Western Asia and Mediterranean regions are considered to be its two distributional centers (Wu \& Li 1982). Ca. 84 species are distinguished in China. Of which, about 70 species are endemic to China, and most species distributed in the Hengduanshan Mountains region (LI \& Hedge 1994). Fujita (1970) suggested that Salvia is the most primitive genus in the family Lamiaceae based on its basic chromosome number 11, the highest basic chromosome number of the family. Wu and LI (1982) considered that salvia is more advanced and specialized group for its floral adaptation to insect visitors.

There are several cytological reports on the genus from Europe, America and Asia (GIll 1970, 1971, 1984; PAtUdin et al. 1975; SARKar et al. 1975; Afzal-Rafit 1976; Reveal and Moran 1977; BhatTACHARYa 1978; KIEF and Van Loon 1978; Haque and Ghoshal 1980; Mercado et al. 1989; Harley and Heywood 1992; Del Carratore and Garbari

\footnotetext{
* Corresponding author: phone: +86-0871-5223625; fax: +86-0871-5150227; e-mail: gongxun@mail.kib.ac.cn or gongx@km169.net.
}

1996). The previous data indicated surprisingly diverse chromosome numbers in the genus. The genus seems to be polybasic, with different groups of species in different parts having polyploid origins. Species distributed in Mediterranean region seem to be mainly characterized by basic chromosome number of $x=7$ (AFZAL-RAFII 1976), those in Europe and Russia by $\mathrm{x}=11$ (Patudin et al. 1975), those in California by $\mathrm{x}=16$ (Epling et al. 1962), those in Mexican by $\mathrm{x}=11$ (Reveal and Moran 1977; Palomino et al. 1986). Although some researchers (GILl 1970, 1984; Haque et al. 1980; Bhattacharya 1978) did chromosome studies on Salvia from the West-Himalayas, the cytological data of the genus from the Hengduanshan Mountains region are still poorly known. Moreover, the Hengduanshan Mountains region is considered to be one center of speciation and diversification of Salvia (Wu and Li 1982). The cytological studies were thought desirable to work out the principal mechanism involving speciation and evolution as well as to find out affinity and phylogeny among the members of the genus. Most alpine plants outside Asia have been found to be diversified via hybridization and polyploidization during Quaternary climatic change (STEBBINS 1984). Cytological information may also help in breeding and horticultural works, for important horticultural and medicinal value of some species of the genus. The main aim of this research is to confirm the basic chromosome number of Salvia and to discuss the 
speciation pattern in Hengduanshan Mountains region.

\section{MATERIALS AND METHODS}

The materials for cytological analysis here were introduced and cultivated in Kunming Botanical Garden (Table 1). Voucher specimens were deposited at the herbarium of Kunming Institute of Botany, Chinese Academy of Sciences (KUN).

For the observations of somatic chromosomes, the growing root tips were pretreated in $0.05 \%$ colchicine solution at room temperature for $3 \mathrm{~h}$, and were then fixed in acetic alcohol (3:1=absolute ethanol and glacial acetic) at $4^{\circ} \mathrm{C}$ for 30 minutes. They were macerated in 1:1 mixture of $1 \mathrm{~mol} / \mathrm{L}$ hydrochloric acid and $45 \%$ acetic acid at $60^{\circ} \mathrm{C}$ for 10 minutes and then were stained and squashed in $1 \%$ aceto-orcein solution before observation.

Karyomorphological observations were made on chromosomes at resting stage and mitotic prophase and metaphase, and their classifications followed TANAKA (1971, 1977). Karyotype formulas were based on the data of measurements of mitotic-metaphase chromosomes from photomicrographs. The nomenclature used to describe the karyotype followed Levan et al. (1964). The classification of karyotype asymmetry followed STEBBINs (1971).

\section{RESULTS}

The chromosomes at resting stage and mitotic prophase as well as metaphase of six species exam- ined were shown in Figures 1. The morphology of the chromosomes at resting stage and mitotic prophase of three species were the same. The characteristics of karyotypes were summarized in Table 2.

In the nuclei at resting stage, several darkly stained heteropycnotic bodies, which were irregularly protruded, were clearly observed. The other region was just very dilutely stained, so the boundary of the bodies was rather distinct (Fig.1: A). Thus, the karyomorphology of the nuclei at resting stage was categorized to be simple chromocentre type.

In the mitotic prophase chromosomes, heteroand euchromatic segments were distinguishable, and heterochromatic segments were mainly distributed in the proximal regions (Fig.1: B). The morphology of mitotic prophase chromosomes was categorized to be the proximal type.

1. S. flava Forrest - This species is endemic to the Hengduanshan Mountains region, and two varieties under this species have been distinguished in China, namely $S$. flava var. flava, and S. flava var. megalantha. S. flava var. falva showed the chromosome number of $2 \mathrm{n}=16$ at mitotic metaphase ranging in length from 1.36 to $2.56 \mu \mathrm{m}$ (Fig.1: C and Fig.2: A) The chromosome complement at mitotic metaphase consisted of 16 submedian-centromeric chromosomes. Karyotype asymmetry was estimated as the STEBbINS' 3 A.

2. Salvia digitaloides var. digitaloides Diels - This species is endemic to the Hengduanshan Mountains region, and two varieties under this species have been distinguished in China. S. digitaloides var. glabrescens is widely distributed in the region with altitudes of 2300 2500 $\mathrm{m}$ in southwest China, and S. digita-

Table 1 - Origin of materials

\begin{tabular}{llll}
\hline \multicolumn{1}{c}{ Taxon } & \multicolumn{1}{c}{ Locality } & Altitude (m) & Voucher \\
\hline Salvia digitaloides var. digitaloides & Zhongdian,Yunnan & 3350 & ZY Yang 02191 \\
S. przewalskii var. przewalskii & Lijiang, Yunnan & 2800 & ZY Yang 02221 \\
S. castanea & Lijiang, Yunnan & 2900 & ZY Yang 02229 \\
S. flava & Zhongdian,Yunnan & 3400 & ZY Yang 02234 \\
S. trijuga & Zhongdian,Yunnan & 3400 & ZY Yang 02110 \\
S. yunnanensis & Eryuan,Yunnan & 2100 & ZY Yang 02112 \\
\hline
\end{tabular}

Table 2 - Chromosome number (2n) and karyotypes

\begin{tabular}{llll}
\hline \multicolumn{1}{c}{ Species } & $2 \mathrm{n}$ & Karyotype & Asymmetry \\
\hline S.flava & 16 & $16 \mathrm{sm}$ & $3 \mathrm{~A}$ \\
S. digitaloides var. digitaloides & 16 & $16 \mathrm{~m}$ & $3 \mathrm{~B}$ \\
S. trijuga & 16 & $8 \mathrm{~m}(1 \mathrm{sat})+4 \mathrm{sm}+4 \mathrm{st}$ & $2 \mathrm{~A}$ \\
S. castanea & 16 & $2 \mathrm{~m}+4 \mathrm{sm}+10 \mathrm{st}$ & $3 \mathrm{~B}$ \\
S. yunnanensis & 16 & $6 \mathrm{~m}(1 \mathrm{sat})+8 \mathrm{sm}+2 \mathrm{st}$ & $2 \mathrm{~A}$ \\
S. przewalskii var. przewalskii & 32 & $24 \mathrm{sm}+8 \mathrm{st}$ & $3 \mathrm{~B}$ \\
\hline
\end{tabular}




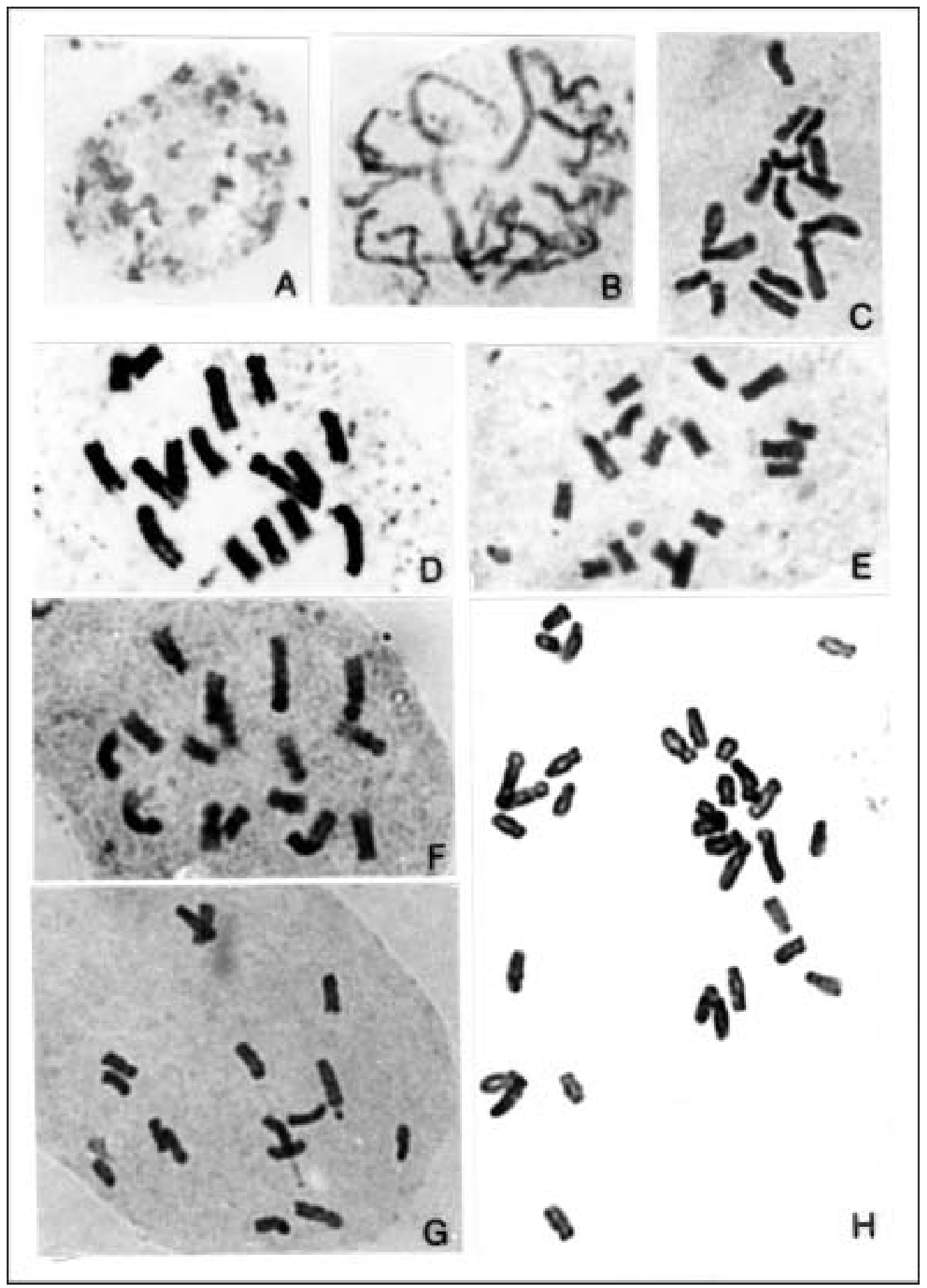

Fig. 1 - A. The resting nuclear of Salvia flava. B. The prophase of S. flava. C-H. The metaphase chromosomes of six species in Salvia. C) S. flava; D) S. digitaloides var. diaitaloides; E) S. trijuga; F) S. castanea; G) S. yunnanensis; H) S. przewalskii var. przewalskii. Bar $1 \mu \mathrm{m}$.

loides var. digitaloides with white tomentulose is a substitute taxon of the former and only distributed in the alpine region with altitudes of 2500 3400 $\mathrm{m}$ in northwest Yunnan province. S. digitaloides var. digitaloides showed the chromosome number of $2 \mathrm{n}=16$ at mitotic metaphase ranging in length from 1.18 to $3.07 \mu \mathrm{m}$ (Fig.1: D and Fig.2: B). The chromosome complement mitotic at metaphase consisted of 
16 median-centromeric chromosomes. Karyotype asymmetry was estimated as the STEBBINS' 3B.

3. S. trijuga Diels - This species is endemic to the area with altitudes of 1900 3900 $\mathrm{m}$ in the Hengduanshan Mountains region. S. trijuga showed the chromosomes number of $2 \mathrm{n}=16$ at mitotic metaphase ranging in length from 1.13 to $2.09 \mu \mathrm{m}$ (Fig.1: $\mathrm{E}$ and Fig.2: C). The chromosome complement at mitotic metaphase consisted of 8 median-centromeric, 4 submedian-centromeric and 4 subterminal-chromosomes, and a satellite was observed on the short arm of one chromosome of the 7th pair. Karyotype asymmetry was estimated as the STEBBINs' 2A.

4. S. castanea Diels - This species is distributed in alpine area with altitudes of 2500 3800 $\mathrm{m}$ in the Hengduanshan region such as Yunnan, Sichuan and Xizang. S. castanea showed the chromosome number of $2 \mathrm{n}=16$ at mitotic metaphase ranging in length from 1.23 to $2.89 \mu \mathrm{m}$ (Fig.1: F and Fig.2: D). The chromosome complement at mitotic metaphase consisted of 2 median-centromeric, 4 submedian-centromeric and 10 subterminal-chromosomes. Karyotype asymmetry was estimated as the STEBBINs' $3 \mathrm{~B}$. The result is different from the previous report that the base chromosome number is 11 (GILL 1984).

5. S. yunnanensis C. H. Wright - This species is only distributed in the area with altitudes of 1800 2900 $\mathrm{m}$ in Yunnan, Sichuan and Guizhou of southwest China. S. yunnanensis had the chromosomes number of $2 n=16$ at mitotic metaphase ranging in length from 1.20 to $1.92 \mu \mathrm{m}$ (Fig.1: $\mathrm{G}$ and Fig.2: E). The chromosome complement at mitotic metaphase consisted of 6 median-centromeric, 8 submedian-centromeric and 2 subterminal-chromosomes, and a satellite was observed on the short arm of one chromosome of the first pair. Karyotype asymmetry was estimated as the STEBBINs' 2A.

6. S. przewalskii var. przewalskii Maximowicz - This species is widely distributed in the western China, and its morphology is various. Four varieties under this species have been distinguished in China based on their corolla color and indumentum on the leaves, namely $S$. przewalskii var. przewalskii, S. przewalskii var. mandarinorum, S. przewalskii var. glabrescens and S. przewalskii var. alba. S. przewalskii var. przewalskii was the tetraploid with the chromosomes number of $2 \mathrm{n}=4 \mathrm{x}=32$ at mitotic metaphase ranging in length from 1.07 to $2.29 \mu \mathrm{m}$ (Fig.1: $\mathrm{H}$ and Fig.2: F). The chromosome complement at mitotic metaphase consisted of 24 submedian-centromeric and 8 subterminal-chromosomes. Karyotype asymmetry was estimated as the STEBbins' 3B. The result is different from that of PATUDIN et al. (1975), which the species from Russia is diploid with a chromosome number of $2 \mathrm{n}=2 \mathrm{x}=16$.

\section{DISCUSSION}

All species studied here shows the same karyomorphological characteristics at resting stage and mitotic prophase. S. przewalskii var. przewalskii is the only tetraploidy species studied here with the chromosome number of $2 \mathrm{n}=4 \mathrm{x}=32$, other species are diploids with the same chromosome number of $2 \mathrm{n}=2 \mathrm{x}=16$.

S. przewalskii and $S$. castanea are eurychoric species. PAtudin et al. (1975) reported that S. przewalskii from Russia is a diploid with a chromosome number of $2 n=2 x=16$, but the result here showed it is the tetraploid with the chromosomes number of $2 n=4 x=32$. Therefore, there are different chromosome-ploidys in $S$. przewalskii in different regions. The result here shows the basic chromosome number of $S$. castanea from Hengduanshan Mountains region is $\mathrm{x}=8$, which is different from $\mathrm{x}=11$ from West-Himalayan region reported by GILL (1984). It is possible that there are two basic chromosome numbers in $S$. castanea at least if the techniques and/or the plant identification were accurate.

The perusal of the literature indicates surprisingly diverse chromosome numbers in the Salvia genus. The genus seems to be polybasic, with different groups of species in different parts having polyploid origins. The Mediterranean group seems to be characterized by $x=7$ (AFZAL-RAFII 1976), those in Europe and Russia by $x=11$ (PATUdin et al. 1975), those in California by $x=16$ (EPLING et al. 1962), those in Mexican by $\mathrm{x}=11$ (Reveal and Moran 1977; Palomino et al. 1986). The result here show that group in Hengduanshan Mountains region seems to be characterized by $x=8$. The species with a basic number of $\mathrm{x}=8$ occur widely in the whole distribution of Salvia but do not occupy a dominant position in other region except in the Hengduanshan Mountains region. The Salvia genus is considered to origin in Mediterranean area and to disperse in the tropical and temperate zones of the Old and New World (Wu \& Li 1982). The diverse basic chromosome numbers in the Salvia genus indicate that the groups of species in the different regions have differentiated independently after dispersal. The flora of the Hengduanshan Mountains region started in late Cretaceous and early Paleogene. The uplift of the Hengduanshan Mountains region caused the semiarid and arid flora of Mediterranean turning to adapt to alpine environments and gradually differentiated and developed in these regions (Sun 2002). Some 


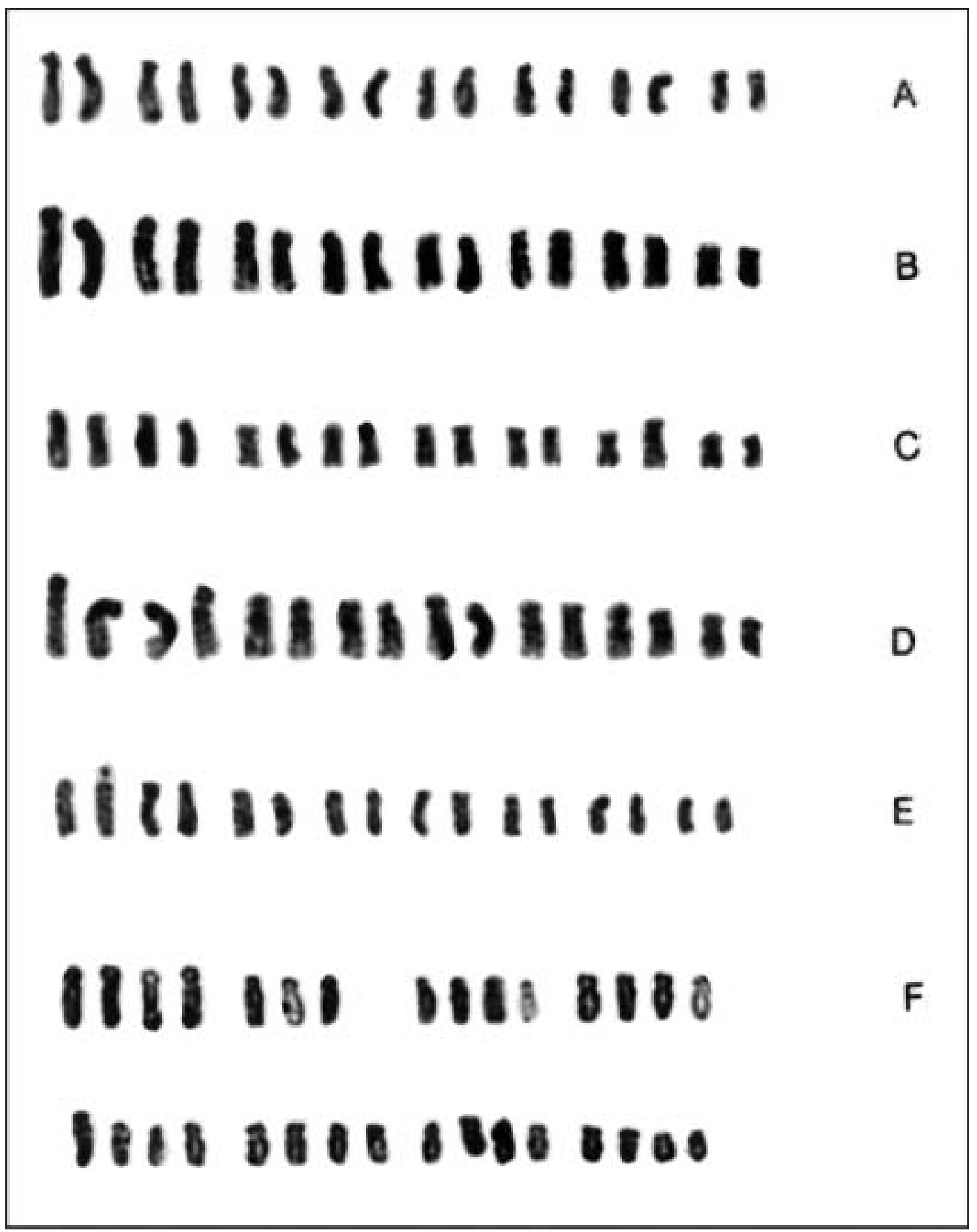

Fig. 2 - The karyotypes on Salvia. A) S. flava; B) S. digitaloides var. diaitaloides; C) S. trijuga; D) S. castanea; E) S. yunnanensis; F) S. przewalskii var. przewalskii. Bar $1 \mu \mathrm{m}$.

Salvia species differentiated and developed in the alpine environments. According to the fact the species with a basic number of $\mathrm{x}=8$ occur widely in the whole distribution and occupy a dominant position in the Hengduanshan Mountains region, it is possible that Salvia species occurring in these regions originated from the species with a basic number of $\mathrm{x}=8$ by the variation of chromosome structure and isolation speciation.

Plants are well known for sympatric and abrupt production of new species through autopolypoidy or allopolypoidy via hybridization at the point of con- 
tact of two existing species (STEBbins 1974; Briggs \& WALTERs 1997). Before starting this study, it was expected that polyploids would prevail in this group based on the following facts. First, all six species investigated here always show sympatric distribution, e.g. S. flava, S.trijuga and S.digitaloides. This distribution usually infers speciation through polyploidy because only this method could result effectively and quickly in the reproductive separation of populations growing together (STEBbins 1971, 1974; GRANT 1981). Second, most species are restricted to alpine habitats in the Hengduanshan Mountains region and facultatively reproduce through both sexual seeds and vegetative rhizomes, whereas the frequency of polyploid species tends to be relatively high in highaltitude region and they usually display this facultative reproduction (StebBINS 1971, 1974; Grant 1981). Third, the Pleistocene climate and topography in the Hengduanshan Mountains region (Li et al. 1995; SHI et al. 1998) should have provided good opportunities for polypoid establishment through hybridization in the contact zones of exiting species and opening new habitats for polyploid vegetative colonization during the advance and retreat of ice sheets and glaciers (GRANT 1981). By contrast, except $S$. przewalskii var. przewalskii is the tetraploid, other five species mainly restricted in alpine habitats are diploid. This fact indicates that sympatric speciation through polyploidization is a minor factor in the early species diversification but that variation of karyotype structure at the diploid level seems to be the predominant feature of chromosomal evolution of this group in the Hengduanshan Mountains region.

Several recent karyological researchers on diversity and speciation of species distributed in the Hengduanshan Mountains region also confirmed this prediction. Ligularia, Cremanthodium, Nannoglottis, Sinacalia and Parasenecio (Asteraceae) are typical alpine genera and endemic to or mainly distributed in the Hengduanshan Mountains region. Karyomorphological studies of these genera have revealed that almost investigated species are diploip species with the uniformity of basic chromosome number and karyotypes in genus (Liv 2000; Liu et al. 2000, 2001; GAO 2001; Gong et al. 2001; LiU 2003; PAN et al. 2004). Karyomorphological study of the Hengduanshan Mountains region endemic Tibetia (Fabaceae) has showed that all three investigated species also are diploid with a chromosome number of $2 \mathrm{n}=16$ and similar karyotypes (NIE et al. 2002). Chromosomal study of some plants from Hohxil region with an average altitude of $5000 \mathrm{~m}$ also showed that all investigated species are diploid (YANG and Wu 1993). Therefore, it is assumed that the species diversity of the alpine plants in Hengduanshan Mountains region has resulted from the great climatic diversity of the area, combined with opportunities for allopartic speciation afforded by repeated fragmentation and coalescence of populations during the uplifting of the Qinghai-Tibet plateau within the last four million years.

Acknowledgements - We thank Professor Li Hsiwen for his identifying species. We are grateful to Dr. Yi Tingshuang for his critical reading of the manuscript and constructive suggestions. This work was supported by grants of Science Foundation of Yunnan Province (2001C0021Q, 2003C0062M).

\section{REFERENCES}

Afzal-Rafin Z., 1971 - Contribution a l'etude cytotaxonomique des Salvia de Turquie I. Bull. Soc. Bot. Fr. 118-69-76.

BhAtTACHARy S., 1978 - Study of some Indian members of the genus Salvia with references to the cytological behavior. Cytologia, 43:317-324.

Briggs D. and Walters S. M., 1997 - Plant variation and evolution, $3 \mathrm{rd}$ edn. Cambridge, UK: Cambridge University Press.

Del Carratore F. and Garbari F., 1996 - Mediterranean Chromosome Number Reports 6 (700-704). Flora Mediterranea, 6: 266-277.

Epling C., Lewis H. and Raven P. H., 1962 - Chromosomes of Salvia, section Audibertia. Aliso, 5: 217 221.

FujITA, YASUJI, 1970 - Evolution of chromosome numbers in the Labiaceae, especially its relation to the classification and phylogeny of the genus Salvia based on constituents of essential oils. Acta Phytotaxon. Geobot., 24:113-121.

Gao T.G., 2001 - A systematic study of the genus Nannoglottis Maxim (Compositae). Unpublished $\mathrm{PhD}$ Dissertation. Beijing: Institute of Botany, Chinese Academy of Sciences.

GILL L. S., 1970 - Cytological observations on WestHimalayan Labiatae: Tribe. Stachydeae. Phyto, 27: $177-184$

Gill L. S., 1971 - Chromosome studies in Salvia (Labiatae) West-Himalayan species. Experientia, 27:596-598.

GILL L. S., 1984 - The incidence of polyplody in the West-Himalayan Labiatae. Rev. Cytol. Biol. Végét. Bot, 7:5-16.

Gong X., Gu Z. J., Lu Y. X. and Zhang C. Q., 2001 The karyotypes of seven species in Ligularia. Acta Botanica Yunnanica, 23: 216-222.

Grant V., 1981 — Plant speciation. New York: Columbia University Press.

Harley R. M., Heywood C.A., 1992 - Chromosome numbers in tropical American Labiatae. In HARLEY and Reynold (eds), Advances in Labiatae Science, pp.221-246. Royal Bot. Garden, Kew. 
Haque M. D. and Ghoshal K. K., 1908 - Karyotypes and chromosome morphology in the genus Salvia Linn. Cytologia, 45:627-640.

Levan A., Fredga K. and Sandberg A., 1964 - Nomenclature for centromeric position on chromosomes. Hereditas, 52:201-220.

Li H. W. and Hedge I. C., 1994 - Lamiaceae. In: Wu Z. Y., Raven P. H. (eds), Flora of China, 17: $195-$ 223. Beijing: Science Press and St. Louis: Missouri Botanical Garden.

Li J. J., SHI Y. F. and Li B. Y., 1995 - Uplift of the Qinghai-Xizang (Tibet) plateau and global change. Lanzhou: Lanzhou University Press.

Liu J. Q., Ho T. N. and Liu S. W., 2000 - Systematic position of Nannoglottis: Karyomorphological data. Acta Phytotaxonomica Sinica, 38:236-241.

LiU J. Q., 2000 - Karyotpes of 4 species in Sinacalia and Parasenecio. Acta Botanica Yunnanica, 22: 447-450.

Liu J. Q., Ho T. N. and Liu S. W., 2001 - Karyological studies on the Sino-Himalayan endemic genus, Cremanthodium (Asteraceae: Senecioneae). Bot J Linn Soc, $135: 107-112$.

LIU J. Q., 2004 - Uniformity of karyotypes in Ligularia (Asteraceae: Senecioneae), a bighly diversified genus of the eastern Qinghai-Tibet Plateau highlands and adjacent areas. Bot J Linn Soc, 144:107-112.

Mercado P., Pamamoorthy T. P. and Palomino G., 1989 - Karyotypes of five Mexican species of Salvia subgenus Calosphace (lamiaceae). Cytologia, 54:605. 608.

Nie Z. L., Gu Z. J. and Sun H., 2002 - Cytological study of Tibetia (Fabaceae) in the Hengduanshan Mountains region of China. Journal of Plant Research. 115:17-22

Patudin A. V., Yurtsev V. N. and Pakaln D. A., 1975- Chromosome number in some species of Salvia L. (Lamiaceae). Вотанический Журиал, 60(4): 529-534.
Palomino G., Mercado P. and Ramamoorthy T. P., 1986 - Chromosomes of Salvia subgenus Calosphace (Lamiaceae), a preliminary report. Cytologia 51: 381-386.

Pan Y. Z., Gong X., and YIn Q., 2004 - The karyotypes of five species in Ligularia. Acta Botanica Yunnanica, 26: 65-72.

SHI Y. F., Li J. J. and Li B. Y., 1998 - Uplift and environmental change of Qinghai-Tibetan in the late Cenozoic. Guangzhou: Guangdong Science and Technology Press.

Stebins G. L., 1971 - Chromosome Evolution in Higher Plants. London: Edward Arnold Ltd.

StebBins G. L., 1974 - Flowering plants: evolution above the species level. Cambridge, MA: Harvard University Press.

Stebbins G. L., 1984 - Polyploidy and distribution of the arctic-alpine flora: new evidence and new approach. Botanica Helvetica, 94:1-13

Sun H., 2002 - Tethys retreat and Himalayas-Heduanshan Mountains uplift and their significance on the origin and development of the Sino-Himalayan elements and alpine flora. Acta Botanica Yunnanica, 24: 273-288.

Tanaka R., 1971 - Types of resting nuclei in Orchidaceae. Bot. Mag. (Tokyo), 84:118-122.

Tanaka R., 1977 - Recent karyotype studies. In: Ogawa Y, et al. (eds.), Plant Cytology. Tokyo: Asakura Shoten.

Vij S. P. and Kashyap S. K., 1976 - Cytological studies in some North Indian Labiatae. Cytologia, 41713 717.

Wu C. Y. and LI H. W., 1982 - On the evolution and distribution in Labiatae. Acta Botanica Yunnanica, 4: 97-118.

YANG Y. P. and Wu S. G., 1993 - Chromosomal reports on some plants of Hohxil region, Qinghai (1). Acta Botanica Yunnanica, 15: 173-178. 